\title{
UNE ONG AU RISQUE DE L'ENGAGEMENT : PARTICIPATION ET COORDINATION DES BÉNÉVOLES, MILITANTS ET SALARIÉS
}

\author{
Vincent BRULOIS ${ }^{1}$
}

Depuis l'origine, Amnesty International France (AIF) se présente comme un "mouvement bénévole et militant». Au fil du temps, l'ONG connaît une évolution raisonnée qui a favorisé une audience et une influence plus forte. Aujourd'hui, cette notoriété s'accompagne d'une professionnalisation de ses structures, marquée notamment par un important recrutement de nouveaux membres comme de nouveaux salariés. Ces évolutions ne se font pas sans heurts. Ainsi, les bénévoles apparaissent en manque de reconnaissance et les militants se sentent parfois dépossédés de leurs prérogatives au profit de salariés perçus comme des «personnes de passage », moins imprégnés de l'histoire, des valeurs et de l'identité du mouvement, en un mot " moins militants ». Nous nous intéresserons donc à la façon dont les changements qui touchent AIF redistribuent les cartes de la participation de chacun au projet associatif en fonction de son statut (bénévole, militant, salarié). À l'heure où les frontières se troublent (travail bénévole, engagement salarié), comment l'ONG parvient-elle à organiser le travail de tous sans désespérer la participation bénévole et l'engagement militant? De quelle façon parvient-elle, tout à la fois, à manager et à ménager les individus?

1 Vincent Brulois est maître de conférences en Sciences de l'Information et de la Communication à l'Université Sorbonne Paris Nord, responsable du master Communication et RH et membre du LabSIC.

Recherches en communication, $\mathrm{n}^{\circ} 52$ - Article publié le 08/09/2021 


\section{Introduction}

Dans les entreprises, il est à présent usuel d'entendre parler d'engagement et de participation. L'engagement du salarié renvoie par exemple à l'énergie développée par l'individu pour exécuter ses tâches, à l'implication consentie pour trouver une solution aux aléas qui surgissent, à la motivation mise pour s'intégrer dans l'organisation en place et à s'adapter aux évolutions qu'elle connaît. Quant à la participation, le terme renvoie notamment à tout un ensemble de démarches et de dispositifs développés, à l'orée des années 1980, par les directions des grandes entreprises pour en promouvoir un nouveau fonctionnement dans un contexte naissant de mondialisation. Dans un dossier sur la participation d'une revue éponyme, Béroud (2013) note la pluralité de sens attribués par les acteurs à cette notion, ce qui en fait à tout le moins un « objet relativement mal défini » (ibid., p. 9), voire une " notion polémique » (ibid., p. 7$)^{2}$.

Si engagement et participation sont donc bien présents dans le monde du travail, le secteur associatif est identifié de longue date comme le « monde de l'engagement» (Simonet, 2006, p. 191). En première instance, l'engagement personnel renvoie à une cause qui dépasse l'individu, une cause à laquelle il n'est pas forcément directement confrontée $^{3}$ et à laquelle il adhère pourtant en conscience. L'engagement associatif fait alors apparaître, si ce n'est un effacement de l'individu devant une cause qui le dépasse, à tout le moins une « idée de dévouement à autrui et d'oubli de soi » (Combes \& Ughetto, 2010, p. 168). La participation de chacun s'effectue alors à l'aune de cet engagement personnel vis-à-vis de l'association, pour prendre part à un « univers de sens en train de se faire » (Cefaï et al., 2012, p. 13).

Créée en 1971, Amnesty International France (AIF) représente la section française de l'organisation non gouvernementale (ONG) Amnesty International (AI). Ce «mouvement $t^{4} »$ international, qui se

2 Pour la défricher plus longuement, on peut se reporter, dans le même numéro de la revue, à l'article d'Antoine Bevort : De Taylor au néo-management : quelle participation des salariés ? Participations, 5(1), 2013, pp. 33-51. Doi : https://doi. org/10.3917/parti.005.0033).

3 Par exemple, le militant antiraciste n'est pas forcément une victime d'actes racistes.

4 Ces verbatim, sans indication d'auteur, sont issus des entretiens que nous avons menés. Il en sera de même pour les suivants. Toutefois, pour une meilleure compréhension du propos, il sera indiqué la qualité de celui ou celle qui parle : bénévole, 
veut dès l'origine « bénévole et militant (salarié), est né dix ans auparavant autour d'une thématique issue de la Déclaration universelle des Droits de l'Homme - l'exigence de libération des prisonniers d'opinion dans le monde -, de trois valeurs fondatrices - neutralité, impartialité et indépendance - et porté par un mot d'ordre clair - exiger la stricte application du droit. Au cours du temps, le « mandat limité et strictement défini » d'AI (de Montalembert, 1980, p. 323) a certes connu un élargissement aux droits économiques, sociaux et culturels en 2001, mais sans perdre de vue pour autant la logique initiale de mettre la défense des droits individuels « à la portée de tous » (ibid., p. 322). Cette évolution raisonnée a ainsi favorisé une audience et une influence plus forte à l'échelle internationale 5 .

Cette notoriété accrue s'est accompagnée il y a peu d'une réorganisation de ses structures, qui s'est traduite par un important développement à la fois de ses ressources humaines (recrutement de salariés) et de ses ressources financières (collecte de dons). Cette réorganisation semble d'autant plus nécessaire que, en parallèle, l'association affiche une volonté de croissance qui se concrétise par une démarche active d'adhésion de nouveaux membres ${ }^{6}$. Au sein d'AIF, ces évolutions marquent le constat que le «vieux modèle d'Amnesty " (bénévole) doit s'adapter à ce nouvel environnement. Dès lors, de quelle façon l'association peut-elle évoluer tout en restant fidèle aux valeurs amnestiennes qui la fondent? Comment offrir aux membres, nouveaux comme anciens, des choix de participation en phase avec les attentes individuelles? Dans quelle mesure un engagement hier « total » (Ion, 1997) peut-il cohabiter avec un engagement aujourd'hui plus " distancié » (ibid.) ou « désimpliqué » (Pierron, 2006, p. 58) ?

À l'aune des évolutions que connaît l'ONG, nous porterons notre regard sur la dynamique organisationnelle à l'œuvre et portée par ses acteurs, qu'ils soient militants, bénévoles, salariés ou encore nouveaux membres. Pour mener à bien cette analyse à la fois sociologique et communicationnelle, nous nous appuierons sur une enquête par entre-

militant ou salarié. En outre, le choix a été fait d'indiquer ces propos d'acteurs, entre guillemets et en italique, afin de bien les différencier des propos d'auteurs, entre guillemets sans italique, et dûment référencés.

5 Cette influence a notamment été reconnue par l'attribution du Prix Nobel de la Paix en 1977 et du Prix des Nations Unies pour les Droits de l'Homme en 1978.

6 «On est passé de 30000 membres en 2013 à 90000 aujourd'hui 》 (salariée), c'està-dire en 2018. 
tien $^{7}$ menée au sein du Secrétariat national (SN) d'AIF à l'initiative de l'ONG. Le résultat en est une parole sollicitée, permettant d'explorer des « faits » concernant « les systèmes de représentations (pensées construites) et les pratiques sociales (faits expériencés) » (Blanchet \& Gotman, 2006, p. 25). Par celle-ci, il nous a été possible de dégager une représentation du contexte d'action ou de travail des acteurs à partir de la position qui est la leur, de percevoir leurs craintes, leurs doutes ou leurs espoirs et, en fin de compte, de percevoir la construction subjective du sens de leur action dans ce contexte de changement. C'est donc dans une analyse discursive ${ }^{8}$ de ces entretiens et une perspective compréhensive de ces discours que nous nous situons. Certes, l'observation directe aurait donné accès aux pratiques, qu'il est toujours intéressant de rapprocher des discours. Mais nous ne l'avons que trop peu fait pour en tirer un quelconque enseignement. Nous nous sommes donc contentés, en quelque sorte, de considérer les individus " pour ce qu'ils sont » et « pour ce qu'ils disent qu'ils sont» (Vermeersch, 2004, p. 684).

Dans ce cadre, notre propos insistera sur la façon dont les changements qui touchent AIF redistribuent les cartes de la participation de chacun au projet associatif en fonction de son statut : bénévole, militant, salarié. Pour ce faire, nous passerons par quatre éclairages : sur la notion d'engagement, sur le développement de l'ONG entre communauté et organisation, sur son articulation autour de plusieurs lieux de vie (engagement, travail), sur son usage de la parole enfin.

725 entretiens semi-directifs longs (une durée d'environ une heure et demie) ont été menés au secrétariat national d'AIF à l'été 2017 auprès de salariés, de militants et de bénévoles, choisis en majorité par la section française de l'ONG. Sans être représentatif, cet échantillon a essayé de tenir compte de la diversité des profils quant au statut (salarié ou membre), à l'ancienneté (nouveaux adhérents, jeunes ou anciens membres), à l'âge (moins de 30 ans, plus de 60 ou entre les deux), à la géographie de l'engagement (Paris, Île de France ou province), ou encore aux responsabilités (membre du Conseil d'administration, d'un groupe local, d'une antenne jeune ou membre individuel). L'enquête a donné lieu à une restitution devant le Conseil d'administration en mars 2018.

8 La méthode de l'analyse discursive est choisie pour l'intérêt qu'elle accorde à la dimension argumentative du discours (Bonnafous \& Krieg-Planque, 2013). Elle m'a permis de mettre en évidence des formes de continuité ou de rupture dans ces discours et de les interpréter (Seurrat, 2014). 


\section{Engagement et participation en milieu associatif}

Selon Becker (1960), l'engagement permet de « rendre compte des lignes d'actions cohérentes d'un individu » (p. 2). Pour Pierron (2006), l'engagement est « envie d'agir », elle-même déclenchée par une « raison d'agir » (p. 53). Nombre de bénévoles ont ainsi avancé ce « besoin d'agir » comme élément fondamental de leur arrivée chez AIF. Mais cette cohérence dans l'action peut n'apparaître que dans la durée. L'engagement, en effet, n'est pas toujours pris « sciemment et délibérément », se formant plutôt « progressivement » (Becker, 1960, p. 8). Becker toujours a parlé de «paris subsidiaires » (ibid., p. 6) successifs pour caractériser des éléments externes à l'action mais qui « engage[nt] l'individu à agir, dans une situation donnée, de façon cohérente » (ibid., p. 12), tout au moins à ses yeux ; des préalables à l'action en quelque sorte. Joule \& Beauvois (1987) ont montré aussi qu'une suite d'actes anodins - des « actes préparatoires »-peut entraîner l'individu vers l'engagement. S'arrêter à la sortie du métro pour écouter des rabatteurs d'AIF, être sensible à leurs arguments et continuer par une réunion d'information, participer aux échanges sur des forums constituent autant d'événements « modestes » aux effets pourtant « engageants » (Bernard, 2015, p. 91).

Si de petits gestes peuvent faire plus que de grandes idées, l'engagement embarque non seulement le corps de l'individu (j'agis), mais également sa raison (pourquoi j'agis). Il est bien action et réflexion. Pierron (2006) remarque ainsi que l'engagement suppose de « mettre quelque chose » de soi « en gage » (p. 53). Tenir son engagement, c'est alors « interroger la justesse de l'idée que l'on a de soi » et l'ajuster avec ses « capacités d'initiative sur le monde » (ibid., p. 61). Cette réflexion se retrouve en écho dans le discours des bénévoles. Pour l'une, c'est le «côté international » qui a guidé son entrée dans l'ONG. Pour l'autre, le déclic a été la lutte pour l'abolition de la peine de mort. Un troisième, plus énigmatique, relie son adhésion à son " histoire » (militant). Une dernière énonce de façon définitive avoir une « indignation » (militante) qui la pousse à agir, hier comme aujourd'hui. $A$ contrario, d'autres bénévoles ont rationalisé leur engagement chemin faisant. "Amnesty m'a permis de me structurer » reconnaît 1'un, «Je n'étais pas de la génération où les femmes travaillaient et se sont émancipées, et Amnesty avait le temps de m'apporter cela » avoue une autre. Se construire en tant qu'individu ou s'émanciper de sa trajectoire biographique, ces deux propos valident par l'exemple que « les indivi- 
dus poursuivent souvent une ligne d'action pour des raisons tout à fait étrangères à l'action elle-même » (Becker,1960, p. 11). Il n'en reste pas moins que l'engagement n'est pas gratuit pour l'individu, il se fait au prix de la « mobilisation de sa liberté » (Pierron, 2006, p. 53), et en premier lieu du temps qu'il donne à l'association pour laquelle il s'engage.

\subsection{Importance du cadre d'action}

Deux enseignements sont ainsi à tirer de cette discussion liminaire. D'une part, l'engagement individuel est toujours situé dans un contexte, et donc encadré par les règles propres à celui-ci. Pour que l'engagement de l'individu devienne effectif, il faut qu'il rencontre un cadre institué (une association par exemple) dans lequel son envie d'agir trouve à s'accomplir. L'engagement rend alors possible la participation à une action ou un projet, créant ainsi des interactions avec d'autres pour sa réalisation. Ces relations, situées dans un cadre précis, sont ellesmêmes encadrées par des règles organisationnelles. Le cadre est donc ce qui transforme l'engagement personnel (ce qui me pousse à agir) en des pratiques sociales (j'agis), une participation active avec d'autres. Mais, tout en permettant aux engagements de se réaliser, le cadre associatif contraint également les individus, pour le moins en imposant des modalités d'action (lettre de soutien et plaidoyer pour AIF) et en coordonnant les campagnes d'actions contre l'envie de certains militants : «Je pousse pour qu'il y ait des actions plus punchy, pas que des pétitions ou de l'écriture de courriers! » Ainsi, «bonne volonté ou rêves d'utopie » trouvent certes "à s'exprimer dans [ces] espaces normés » (Nicourd, 2007, p. 2), mais ceux-ci délimitent aussi la participation (ce que je peux faire). L'engagement initial se trouve donc fixé par des dispositifs structurés et structurants : "Si l'engagement sans l'institution est inefficace, l'efficacité de l'institution sans l'engagement se fait routinière » (Pierron, 2006, p. 57). En outre, si aujourd'hui, existent toujours en milieu associatif des « régulations communautaires » riches et fortement collectives, s'y développent aussi « des fonctionnements de plus en plus rationalisés qui rencontrent quelques limites dans les modes de mobilisation des ressources bénévoles » note Nicourd (2007, p. 5). Ce premier enseignement fait donc apparaître que de l'engagement à la participation, on passe d'une dimension individuelle à une dimension collective, notamment à travers le mode d'organisation des engagements dans un cadre constitué : "On a commencé dès la première réunion à préparer une action. Ça m'a marqué. » (militant) 


\subsection{Personnalisation de l'engagement}

Second enseignement d'autre part, l'engagement individuel peut évoluer sur la durée. Ainsi, la montée de l'individualisme semble aller à contre-courant de l'engagement associatif ou tout au moins du militant : « Ce qui dissuade » aujourd'hui « dans la figure du militant, ce n'est pas son engagement mais la soumission de l'individuel à l'efficacité collective » (Pierron, 2006, p. 59). Hier, quand on adhérait à AIF, «on $s$ 'engageait à faire trois lettres de soutien par mois » (bénévole). Il faut accepter désormais qu'il y ait des adhérents « de passage », actifs «à un moment, qui ne le seront plus [ensuite], mais qui peuvent revenir plus tard » (salariée). Bref, il faut accepter la «diversité des formes d'engagement » (salarié) et donc des modalités de participation. Cela conduit l'ONG à penser des participations individuelles multiformes dans le temps (participations ponctuelles versus régulières) comme dans 1'espace (présence physique sur le terrain versus présence virtuelle sur les réseaux), des participations à la carte en quelque sorte : "Mon bénévolat, il est important pour moi ; mais il doit entrer dans mon emploi du temps » (bénévole). Tout se passe comme si l'individu était plus dans l'attente d'effets pour soi, quasi immédiats et qui «n'engage à rien » sur la durée (Pierron, 2006, p. 58) : s'engager dans une association, participer avec les autres, mais « si je veux et quand je veux ${ }^{9}$ » : "Je n'ai pas le projet de m'impliquer de manière régulière. Entre le travail, les amis, ma compagne... c'est bien rempli ! Je préfère m 'impliquer [...] pour des actions ponctuelles. ॥ (nouveau membre) Est-ce le signe d'un engagement plus désimpliqué ou plus distancié ? On peut noter en tout cas que la plupart des individus sont toujours à la recherche d'un «sens pour soi, pour les autres et avec les autres » (Nicourd, 2007, p. 5). L'engagement peut connaître aujourd'hui des allures moins engageantes car sa formalisation dans des institutions aux codes bien précis apparaît plus contraignante qu'hier, comme si la « part d'impulsion initiatrice » qui lui est attachée « dispara[issait] et perd[ait] de sa radicalité dans la lourdeur des institutions qui le mettent en œuvre » (Pierron, 2006,

9 En 2002, Demoustier notait une transformation des engagements bénévoles. La recherche d'un « résultat concret, immédiat », notamment, tend à " privilégier certaines actions dont les effets sont visibles et où l'apport personnel est plus perceptible » (Demoustier, 2002, p. 106). Ce que Worms (repris par [Demoustier, 2002, p. 106]), traduisait d'une formule : «On s'investit plus intensément dans l'action mais moins dans l'association " (L'individu, défi et chance pour les associations. Projet, 264, hiver 2000-2001). 
p. 57). Le problème qui se pose alors aux associations est de savoir comment retrouver la fraîcheur de cette impulsion dans des dynamiques organisationnelles et communicationnelles adaptées à l'époque qui est la nôtre?

C'est donc moins la question de l'engagement que celle de la participation aujourd'hui que se pose AIF pour ses membres. La volonté des individus de s'engager chez AIF est toujours présente, preuve en est la multiplication par trois des adhérents en cinq ans. Mais il faut encore «les convaincre de rester avec nous et de participer 》 (salarié). Cela est d'autant plus important qu'AIF se revendique bien comme un mouvement de bénévoles et de militants. Force indéniable, c'est aussi un «sacré travail » (bénévole) que de coordonner la participation de chacun au mouvement amnestien. En effet, puisque le bénévolat relève d'une " démarche non rémunérée » et donc " dépourvue de tout caractère d'obligation » (Vermeersch, 2004, p. 682), dès lors le bénévole est libre de participer comme il l'entend. D'un côté, ayant envie d'agir, les nouveaux membres veulent pouvoir agir. Dans ce sens, la manière nouvelle dont ceux-ci s'approprient le cadre est recherchée par AIF : « la force de [leur] engagement, c'est [leur] créativité, [leur] fantaisie », «ce qu'on veut, c'est qu'ils agissent» (salariée). Mais d'un autre côté, cette nouvelle manière de participer bouscule les fonctionnements habituels et les codes sociaux établis. Leur participation est voulue, souhaitée, encouragée, mais elle ne peut se faire selon la direction élue d'AIF que dans une structure adaptée, repensée, plus souple, nouvelle structure qui a pour conséquence de remettre en cause les équilibres internes.

\section{AIF entre force de sa communauté et nécessaire organisation}

AIF est un «mouvement dans lequel on débat » (militant). Ce principe explicite se double d'un processus applicatif : "On débat, puis on prend des décisions. Ensuite, on applique ces décisions. 》 (militant) Forte d'une culture singulière, AIF porte l'image d'une ONG utile car indépendante, efficace car concrète, influente car internationale. Ainsi, son crédo est clair - «des Hommes qui s'engagent pour des Hommes » (militante) - et ses modalités d'action reconnues - lettre de soutien, plaidoyer et pétition. La pérennité de cette ligne de conduite explique, encore aujourd'hui, l'attraction qu'exerce l'ONG sur l'opinion publique. 
Toutefois, cette notoriété, on l'a vu précédemment, a attiré de nouveaux membres et oblige l'association à se redéfinir en interne (comment accueillir, comment acculturer à la culture amnestienne, comment intégrer ?) et à s'interroger sur les acteurs qui y agissent (qu'est-ce qu'un militant, comment transformer un membre en militant ?) ou qui y interagissent (un bénévole fournit-il un travail, un salarié peut-il être engagé ?). AIF s'agrandit et il faut « savoir accueillir les nouveaux venus » (salariée), leur faire une place dans le fonctionnement, développer de nouveaux processus organisationnels et réinventer des dispositifs de communication. Bref, « il faut s'adapter au nombre de membres qu'on recrute » (salarié). Dès lors, la croissance rapide des effectifs constitue un défi. Somme toute, ce défi est assez banal car nombre d'organisations y sont confrontées. En ce qui concerne AI, de Montalembert (1980) notait déjà à l'orée des années 1980 « l'invention de relais nouveaux » afin « d'éviter qu'un fossé ne se creuse à la longue entre les membres de l'organisation, rompus à son discours et à ses méthodes, et le public des sympathisants dont la participation ponctuelle mais massive donne son relief à l'action du mouvement » (p. 330). C'est justement ce à quoi est confrontée la section française d'AI depuis 2013 avec sa volonté de développer la "participation à la vie militante et démocratique de l'organisation »: comment mieux prendre en compte les attentes des nouveaux membres sans désespérer les anciens ? Quels relais inventés entre bénévoles et salariés afin de maintenir " une efficacité opérationnelle très forte et une vie démocratique intense » (militant) ?

Fort de cette volonté, s'est fait jour la nécessité de structurer l'association, de développer des modes d'organisation du travail et des modalités de gestion des individus, en un mot, une règle - ou à défaut une régulation - acceptable par tous pour agir de façon organisée en " communauté unie autour d'un projet commun » (Hély \& Simonet, 2013). À la fois communauté et organisation, l'ONG est doublement bousculée par cette nouvelle dynamique organisationnelle. Reynaud (1993) a montré qu'un groupe d'individus est en capacité d'agir de concert « dans la mesure où il accepte une régulation »; et « dans la mesure où il accepte une régulation commune, il constitue une communauté ». Dans ce sens, la régulation organise le groupe et constitue la communauté en facilitant son action. Mais il ajoute que chaque acteur produit sa régulation et que, dès lors, « la rencontre des acteurs ou leur vie en commun est aussi la rencontre de deux ou plusieurs systèmes de règles ", comme si co-existaient plusieurs mondes sociaux dans ce 
monde commun (ibid.). Dans le cadre de l'entreprise, Sainsaulieu ${ }^{10}$ a montré que les logiques d'action au travail découlent des caractéristiques propres à chaque entreprise et que leur cohérence génère une identité et une culture communes. D'une part, chaque entreprise est donc un monde social en soi $^{11}$, mais en outre chaque entreprise comprend plusieurs mondes sociaux singuliers. L'entreprise est vue comme un système social vivant, ancré dans une histoire collective et des histoires individuelles, qui tend à faire société. Il convient alors que les modalités d'organisation et de gestion tiennent compte de ces relations singulières. S'inscrivant dans ces propos mais tournant son regard sur les associations, Laville (2013) relève l'importance d'avoir « une adéquation entre l'histoire qui traverse l'association et les méthodes qui sont mises en œuvre en son sein $»$. Les processus de gestion sont toujours contextualisés et plus une association se connaît elle-même, "plus elle a des chances de choisir un chemin pertinent pour ses membres » (id.). À travers les propos de ces membres, la gageure pour AIF semble alors de conserver son « esprit associatif » (Hély \& Simonet, 2013) tout en se développant comme une "entreprise associative » (Hély, 2010, p. 207), reliant à la fois la communauté qu'elle est et l'organisation qu'elle doit devenir.

Pour les associations, la dynamique organisationnelle se traduit souvent par un recrutement de salariés, plus ou moins important en quantité - pour faire «tourner la boutique » (militante) -, mais assurément important en qualité - compétences à faire valoir. Cette « montée en compétences » est d'ailleurs reconnue comme « indispensable, chez les salariés et les bénévoles » (militante). Ce n'est pas nouveau. Bernardeau-Moreau \& Hély (2007) par exemple ont déjà montré que la participation, toute bénévole qu'elle soit, requiert pourtant de plus en plus de compétences afin d'occuper les postes sur les fonctions supports qui se développent dans les associations. Si l'activité associative mobilise toujours la conviction des individus, elle mobilise désormais aussi pour une bonne part la qualification acquise par la formation initiale comme les compétences acquises par l'expérience professionnelle. C'est cet

10 Voir L'Identité au travail (1977, Paris : Presses de Sciences Po), puis Sociologie de l'entreprise : organisation, culture et développement (1997, Paris : Dalloz et Sciences Po Les Presses).

11 Dès 1994, il remarquait ainsi, avec Piotet, que « le risque est aujourd'hui de parler de l'entreprise de manière générique » (Méthodologie pour une sociologie de l'entreprise. Paris : Sciences Po Les Presses). 
état de fait qui a provoqué et provoque encore chez AIF de profonds remous dans les relations internes entre militants, bénévoles et salariés.

\section{AIF comme lieu d'engagement et lieu de travail}

L'exemple d'AIF, sans être emblématique de l'ensemble des associations, nous aide à comprendre de quelles façons l'évolution de ses processus organisationnels bouleverse les «équilibres fondamentaux » de l'association (militant), génère des interrogations parmi ses membres, crée des remous, provoque des réactions, voire oblige à inventer de nouvelles manières d'agir. Ces processus, intervenant au sein d'une association reposant sur le primat de l'engagement bénévole, bouscule l'équilibre entre la structure opérationnelle et la structure représentative de l'ONG : «Les premiers salariés qui ont été embauchés l'ont été au service des bénévoles et c'étaient des secrétaires. Aujourd'hui, [...] on embauche des gens qui ont des Masters. " (salariée) La recherche de compétences spécifiques risque de cantonner « les bénévoles non qualifiés à des tâches d'exécution » (Demoustier, 2002, p. 107) par manque de compétences ad hoc. Tout se passe comme si la notoriété renforcée d'AIF à l'extérieur, conduisait l'ONG à un niveau d'expertise accrue en interne, une complexité plus grande de l'activité, une organisation plus poussée de l'action, la poussant à la mise en place de processus managériaux appropriés.

Ce renversement est plus ou moins bien vécu par les bénévoles. Les militants surtout se sentent parfois dépossédés de leurs prérogatives, au profit de salariés, perçus comme des «personnes de passage » (salariée), moins imprégnés de l'histoire, des valeurs, de la culture et de l'identité du mouvement, moins impliqués, en une formule «moins militants » (salariée). C'est que la vie associative possède des caractéristiques singulières, tenant au fait qu'une association est avant tout la réunion d'individus divers, présents pour des raisons différentes mais, pour autant, présents pour agir ensemble autour d'un projet commun. La « compétence particulière » des associations est justement de « pouvoir transformer des énergies individuelles en capacité d'action collective » (Laville \& Sainsaulieu, 1997). Dans ce sens, AIF est très dépendante des individus qui la composent, de la qualité de leur participation comme de la force de leur engagement. Si cet engagement envers le projet collectif s'atténue, l'association risque de péricliter. Quelle que soit le point d'entrée dans l'ONG (par le bénévolat, le militantisme ou le salariat), l'inscription dans la durée est le fait de la reconnaissance 
d'un « bien commun partagé » (Buccolo et al., 2014, p. 5) pour lequel il convient de se battre, de lutter ou de faire quelque chose (donner de son temps, organiser l'action, faire un choix professionnel), pour le moins de s'engager. Pour favoriser et maintenir la participation de tous, l'association doit leur offrir une expérience riche de construction de leur propre identité, où ils se reconnaissent et où ils sont reconnus. Cette recherche d'expérience comme cette reconnaissance peuvent prendre des formes diverses.

\subsection{Bénévoles et salariés : des raisons d'agir ensemble malgré les difficultés}

Bénévoles et salariés, par exemple, agissent selon deux modes de régulation a priori bien distincts. Le salarié est là pour ses compétences, le bénévole est là pour le temps qu'il donne. Pour autant, dans le fonctionnement quotidien du SN, le travail à faire prime sur le statut des individus qui sont en relation : les uns et les autres doivent agir de concert et coopérer. Mais cette coopération est rendue difficile, au sein même de la relation de travail, par le fait que « le temps des bénévoles $n$ 'est pas le temps des salariés » (militante). En outre, si certains salariés reconnaissent dans les bénévoles des " collègues à part entière 》, certains militants ${ }^{12}$ a contrario ont du mal reconnaître la légitimité du travail salarié : "Les salariés, ce sont nos salariés d'une certaine manière, c'est nous qui les employons, c'est le mouvement qui les emploie »! De fait, le dialogue est nécessaire mais complexe. Le salarié est dans une relation professionnelle avec l'association alors que le militant est dans une relation de bénévolat. Le premier est régi par un contrat explicite - de travail - avec une valeur juridique alors que le second agit dans le cadre d'un contrat implicite sans valeur juridique, mais avec une valeur morale. Pourtant, les deux ont des comptes à rendre à l'ONG dans laquelle ils agissent : «Si on part du principe que, parce qu'on est bénévole, on n'a pas de compte à rendre, que c'est déjà bien qu'on soit là et qu'on donne du temps, ça va pas!» (militante) Il faut considérer que «le bénévole, tout militant qu'il est, [...] est aussi inscrit dans un

12 Pour être plus précis, il conviendrait même de différencier plus clairement le bénévole du militant. Ainsi, «un militant est forcément bénévole ; alors qu'un bénévole n'est pas forcément militant » (militant). Dans la même veine, Lyet (repris par [Demoustier, 2002, p. 103]) identifie le bénévole comme un « militant aux ambitions modestes » (L'Organisation du bénévolat caritatif. Paris : L'Harmattan, 1997). 
processus de travail en commun » (salarié). Ainsi, le militant s'inscrit dans un collectif d'actions organisé. Il ne fait pas ce qu'il veut, il doit rendre des comptes puisqu'il a décidé de s'engager. En une formule, son engagement n'est pas gratuit.

Organiser le travail en association n'est donc pas chose facile. Cela n'a rien d'évident pour les membres de concevoir l'association comme un lieu régi par une régulation de contrôle émanant d'une structure hiérarchique. Pourtant, aujourd'hui, il apparaît que les associations sont aussi des lieux de travail. Chaque jour, au SN, est opérée « une affectation d'individus sinon à des postes, du moins à des tâches » (Combes $\&$ Ughetto, 2010). Preuve d'une organisation en place, cette affectation permet à l'ONG d'effectuer sa production d'expertises, d'interventions et d'actions. Autrement dit, toute action collective, pour reprendre Reynaud (1993), n'est possible que parce qu'il y a agrégation de pratiques individuelles en pratiques réglées. Cette régulation s'impose aux acteurs individuels, leur impose une obligation même si elle repose aussi sur leur consentement.

Monde de communication (on y débat), AIF est donc aussi un monde de travail qui s'organise, qui met en place un «nouveau management » qui tend à faire évoluer les comportements, notamment la «responsabilisation » des salariés comme des bénévoles (salariée). Ce développement nécessite d'étoffer le $\mathrm{SN}^{13}$ et implique de mieux définir le territoire d'action de chacun. Bref, le SN est bien un espace de travail et l'intensité de son fonctionnement rythme l'activité des salariés, des bénévoles comme des militants, revisitant le rôle de chacun et les relations avec autrui.

\section{2. À la recherche de reconnaissance et de sens}

Cette problématique bénévoles/salariés, spécifique aux associations, prend ici une dimension importante en raison de la taille et de l'histoire d'AIF. Dans un tel contexte, nous pouvons tirer deux enseignements conclusifs, concernant chacun des deux groupes. D'une part, les bénévoles (et les militants) découvrent ainsi que leur lieu d'engagement est aussi un lieu de travail. Découragés par ces évolutions, certains se démobilisent, vivent mal les tensions, font l'expérience de la

13 Fin 2017, il y avait déjà plus de 80 salariés et près de 90 l'année suivante. Cette équipe de salariés s'ajoute aux quelque 300 bénévoles qui donnent régulièrement de leur temps au Secrétariat national. 
frustration inhérente au milieu professionnel, recherchent de la « reconnaissance » (bénévole). D'une certaine manière, ils découvrent que leur « liberté est inscrite dans des contraintes » modifiant leur rapport à l'association : " l'altruisme se heurte à la recherche de gratification, la confiance fait place à la responsabilisation, la bonne volonté doit se confronter à la professionnalisation » (Demoustier, 2002, p. 109). Mais d'autres voient dans ces évolutions des possibilités nouvelles - « Si je pouvais gagner ma vie en faisant ce que je fais bénévolement, ce serait bien » (bénévole). Par ce propos, cette bénévole croit en une cause dans laquelle elle s'engage déjà aujourd'hui et pour laquelle elle est prête à travailler demain, en tant que salarié, passant d'un travail non rémunéré à un travail rémunéré. De sorte, non seulement elle identifie son bénévolat à une expérience de travail, au même titre que d'autres, mais recherche aussi un travail qui ait du sens.

Quant aux salariés, à la recherche d'un travail qui fasse sens, ils insistent pour la plupart sur le fait que leur lieu de travail est aussi un lieu d'engagement. Pour l'une, c'est par «foi » et «conviction » qu'elle travaille chez AIF. Pour un autre, c'est un " projet de vie ». Pour tous, c'est un choix, bien plus qu'une opportunité, de travailler dans le secteur associatif, pour une recherche de « sens ». AI agit comme un élément fédérateur. Ils ne travaillent pas uniquement chez AIF, ils font partie du mouvement. Dans ce sens, beaucoup se considèrent comme engagés, l'une avoue aussi militer alors qu'elle est salariée. Dans ce contexte, tout laisse penser qu'un « engagement salarié » existe bel et bien à l'instar de « l'engagement bénévole » (Hély, 2010). Si ce n'est leur travail lui-même, la raison pour laquelle ils le font agit comme une puissante source de motivation, leur apportant une nécessaire satisfaction morale.

L'ONG peut certainement s'appuyer sur cette double appartenance, lieu de travail et d'engagement, pour rapprocher les deux groupes. Il n'en reste pas moins que le travail en commun reste encore difficile. Communauté, AIF est aussi une organisation. La crainte persiste que la remise à plat de cette structure opérationnelle affaiblisse le rôle des bénévoles et des militants. Nous l'avons vu, la légitimité de l'organisation, à travers ses règles et ses procédures, ne va pas de soi pour certains militants. Pour autant, cela n'a pas empêché AIF de repenser sa dynamique organisationnelle. S'il a été imposé, ce choix n'en a pas moins été discuté dans des débats souvent vivants et virulents. 


\section{AIF comme lieu de débats}

Créé il y a près de 50 ans, AIF cherche ainsi à faire évoluer son projet participatif tout en s'appuyant sur son projet associatif. Aujourd'hui, l'ONG se trouve confrontée à une dynamique qui produit du changement sur quatre niveaux : modification de son rapport à l'environnement, qu'il soit adaptatif (élargissement de la mission) ou volontaire (recrutement de nouveaux adhérents), renforcement de la structure et modification du cadre de travail, complexification des relations internes (entre bénévoles, militants et salariés), modification du rapport de chacun à l'association redéfinissant engagement individuel (pourquoi AIF plus qu'une autre ONG ?) comme participation active à la vie de l'association (comment ai-je ma place dans le fonctionnement d'AIF ?). $\mathrm{Du}$ fait de cette dynamique, les frontières se troublent : on observe une « approche professionnelle du bénévolat » (Demoustiers, 2002, p. 106) en même temps qu'une vie militante du salarié. Dans ce contexte prometteur mais incertain, l'ONG cherche alors tout à la fois à manager et à « ménager » les individus (Combes \& Ughetto, 2010, p. 170), en tout cas à faire vivre une conversation interne à l'instar de ce que Giroux et Giordano (1998) ont identifié.

Les "ateliers de la controverse $»^{14}$ en sont un exemple. Espaces d'échanges, mis en place en 2016, afin d'assouplir les tensions inhérentes à la mise en place de la nouvelle organisation, ils ont constitué une réponse pour prendre à bras-le-corps la relation salariés/bénévoles. L'objectif était d'y parler de ce qui devait être " ajusté » (militante) entre les uns et les autres. La démarche était d'autant plus nécessaire que de plus en plus de bénévoles étaient en manque de reconnaissance, l'exprimant à travers un besoin de "paroles [et d']encouragements». Apparait ici le fait que, tout se passe comme si, par leur engagement au sein d'AIF, les bénévoles cherchent à partager des affects et des émotions ; et cela ne se fait pas sans frictions : "Quand on est engagé, on est dans les égo qui parlent à fleur de peau » (salariée).

Compréhensible, cette recherche de reconnaissance est aussi paradoxale. D'un côté, en effet, elle apparaît surprenante. Après tout, le bénévole ou le militant ne font pas dépendre leur engagement d'une quelconque reconnaissance accordée par d'autres. Ils s'engagent pour des raisons personnelles, à la recherche d'un sens pour soi. Leur moti-

14 Malheureusement, je n'ai pas pu les observer in situ. Ce que j'en sais a été obtenu presque par inadvertance, au détour des entretiens. 
vation est guidée par deux valeurs : l'éthique - qui renvoie à la raison d'agir - et le plaisir - qui renvoie à l'envie d'agir - «Militer, [...] c'est aussi un [...] plaisir » (militante). Et la conciliation de ces deux valeurs constitue le « cœur de l'action bénévole » (Vermeersch, 2004, p. 687), chacun choisissant les « variations mélodiques de son engagement » en fonction du temps qu'il peut et veut donner (ibid., p. 690). D'un autre côté, cette recherche de reconnaissance est aussi normale. Après tout, travailler n'est pas qu'un acte rationnel et met en évidence une dimension affective liée au travail ${ }^{15}$. Le bénévole, agissant sur un lieu d'engagement qui devient aussi un lieu de travail, se trouve alors confronté à cette dimension affective du travail. Tout comme le salarié, il a des attentes à la fois en termes de réalisation de soi et de reconnaissance par autrui. Son don - de temps, d'énergie, d'attention - n'est pas gratuit, il en attend - implicitement ou explicitement - des " gratifications plus larges que celles liées à l'accomplissement d'un éventuel "devoir moral" » (ibid., p. 682). Dans quelle mesure alors l'engagement dans le travail (conditionné à la reconnaissance par autrui) entre-t-il en conflit avec l'engagement militant (indépendant de la reconnaissance d'autrui, conditionné au plaisir d'agir)?

Dans ce contexte, les ateliers de la controverse nous semblent significatif des médiations mises en œuvre au sein d'AIF, dans l'objectif de créer un espace communicationnel d'expression des affects, de libération et de canalisation de la parole sur le travail, de régulation des tensions entre militants et salariés et, partant, des conflits internes provoqués par les relations de travail : «Il ne faut pas éluder les choses, [...] il faut exprimer les désaccords » (salarié). En son temps, BlochLainé (1963) avait noté que «la contestation [allait] de pair avec la participation. [...] On s'explique au lieu de se contraindre. » C'est dans ce sens qu'AIF a mis en place ces ateliers ; afin de créer un espace où la parole est possible, un espace où, par la parole, il serait possible d'ajuster l'organisation afin d'améliorer cette relation de travail. On ne saurait mieux dire que l'organisation qui en résulte est bien un « agencement communicationnel » qui cadre et coordonne les activités collectives (Hachour, 2011, p. 196). Loin d'être anodins, ces échanges entre militants et salariés contribuent « à maintenir, à ajuster et à compléter l'organisation dans laquelle ils agissent » (Lacoste, 2000, p. 202). Le

15 Méda et Vendramin ont ainsi montré que le salarié français avait, plus que d'autres, des « attentes expressives à l'égard du travail, ou encore des attentes symboliques, subjectives, réflexives et sociales » (Réinventer le travail. Paris : PUF, 2013). 
langage est une dimension essentielle des interactions sociales entre individus. Leur participation à la vie de l'association dépend de leur capacité à se comprendre et à s'entendre sur les règles de vie au travail, règles qui sont de l'ordre d'un langage commun. Au sein du SN, il s'agit de casser les statuts entre individus et les silos entre pôles afin de "faire en sorte qu'une collaboration se manifeste » (salarié). Au sein d'AIF, plus largement, il s'agit de générer un dialogue permanent afin de faire association comme on fait société ${ }^{16}$.

L'exemple de cette ONG nous montre bien que, participer, c'est « apprendre à maîtriser [...] des manières de voir et de dire, des activités de coopération et de communication » (Cefaï et al., 2012, p. 13). Et cet effort « ébranle les repères de l'affectivité, de la sensibilité et de l'imagination » car c'est un " processus [...] d'expérimentation autant que de discussion » (ibid., p. 13). Dans ce sens, la participation est alors à rapprocher de ce que Hirschman (1970) nomme Voice. Cette participation par la prise de parole cherche à « modifier [...] les manières de faire de l'organisation considérée » (ibid.). Il l'oppose à la défection ou Exit qui est « perdre la possibilité de prendre la parole » (ibid.). En outre, faire défection ne peut être qu'une solution de dernier recours car c'est rompre son engagement. L'engagement représente ainsi une relation en mouvement entre les bénévoles et l'association : les premiers interrogent la seconde en continu (Voice) sur ce qu'ils obtiennent en retour de leur don, réinterrogeant ainsi la fermeté de leur engagement vis-à-vis de celle-ci (Exit). L'engagement individuel constitue bien un enjeu identitaire pour la réalisation duquel les associations donnent aux bénévoles « des moyens de penser son rapport dans le monde et au monde » (Havard-Duclos \& Nicourd, 2005, p. 71). Ainsi, AIF ne peut espérer maintenir l'engagement des bénévoles et réguler leur participation que parce qu'elle parvient à répondre à leurs attentes identitaires. Il convient alors de les ménager, quelles que soient leur qualification, leurs compétences, leur savoir-faire.

Tout le travail d'AIF est donc d'organiser les « règles de la conversation » (Giroux \& Giordano, 1998, p. 148), les « règles de la vie commune au travail » (Ferreras, 2007) en son sein, afin d'arriver à s'expliquer sans se confronter.

16 «C'est compliqué d'essayer de [...] faire du lien social avec tout ces gens qui disent qu'ils ne se connaissent pas parce qu'ils sont jamais là au même moment » (salariée). 


\section{Conclusion}

À l'instar d'autres associations, AIF cherche à évoluer avec son temps dans l'environnement qui est le sien, tout en s'appuyant sur sa culture et son identité. Elle y réussit bien, semble-t-il, si l'on se fie à sa notoriété actuelle et à sa capacité d'attraction. Mais dans les deux cas, cette adaptation à l'environnement l'oblige à interroger ses modes d'action, de communication et d'organisation. Raison pour laquelle il était intéressant de porter le regard sur la dynamique organisationnelle à l'œuvre en son sein et sur les acteurs qui la font vivre, qu'ils soient militants, bénévoles ou salariés. En conclusion de notre propos, force est de constater que des évolutions sont en cours, au moins sur quatre points.

D'abord, l'engagement « total » (Ion, 1997) - caractérisé par l'adhésion à une structure pour laquelle l'individu est prêt à sacrifier son individualité au profit du collectif - évolue vers un engagement « post-it » (id.), où l'individu s'investit dans le temps court de l'action mais moins dans le temps long de l'association. En outre, ces actions ne peuvent s'épanouir que dans un cadre institué qui leur donne cohérence et force. Mais ce cadre doit lui-même évoluer pour accueillir les nouveaux membres et leurs multiples formes d'engagement. Entre fraîcheur de l'engagement individuel et nécessaire formalisation des actions en un cadre institué, l'ONG avance donc sur une ligne de crête.

Deuxième point, du fait de son succès, la communauté AIF (construite dans le temps long) s'agrandit obligeant l'organisation AIF à s'adapter en intégrant les nouveaux membres et leur désir de participation. Renforcée et repensée, la nouvelle structure interroge toutefois les équilibres internes entre bénévoles, militants et salariés. Là encore, l'ONG semble avancer sur un fil, tentant de conserver ses « valeurs amnestiennes » (militante) et son fonctionnement associatif - garant de la communauté qu'elle est - tout en se développant comme l'organisation qu'elle doit être, au risque de devenir une « entreprise associative » (Hély, 2010).

Cette observation nous mène vers une troisième, où l'on constate que le travail bénévole se développe tout autant que l'engagement salarié. Si le premier relève de l'organisation qui s'étend, le second dénote une communauté qui persiste. De l'un à l'autre, ce double développement occasionne des interrogations personnelles quant à sa place et son rôle dans le collectif. Ainsi, plus AIF se restructure, plus le militant se sent (à tort ou à raison) dépossédé de ses prérogatives au profit des salariés. Pour sa part, le salarié, bien inscrit dans la nouvelle organisa- 
tion, cherche toutefois du sens à son travail en recherchant une place dans la communauté militante. Le bénévole, quant à lui, faisait déjà don de son temps à la communauté, mais doit désormais aussi rendre des comptes sur son travail à l'organisation. Les trois sont en quête de reconnaissance.

Enfin, monde de travail en quête de compétences, AIF n'en reste pas moins un monde de communication dans lequel la parole s'échange, au risque de la controverse sans fin, mais toujours au profit d'une conversation interne foisonnante. Communauté au fonctionnement démocratique, l'ONG a toujours été un espace où l'on prend la parole plutôt qu'on ne la donne. Les ateliers de la controverse en sont un exemple significatif. Ils ont permis d'activer un débat sur le contexte organisationnel (quelle est ma place et quelles sont mes relations avec autrui ?), mais aussi sur les dimensions identitaire (qui suis-je, simple bénévole, salarié ou militant ?) et sociale (quelle est la reconnaissance de ce que je fais ?).

Lieu d'engagement, lieu de travail, lieu de débats, AIF est donc un monde social complexe composé de groupes sociaux singuliers. Les processus organisationnels en cours bousculent l'équilibre entre les structures opérationnelle et représentative de l'ONG, tout autant que leurs acteurs. Les changements qui touchent AIF redistribuent les cartes de la participation de chacun au projet associatif ; et ce n'est pas rien si l'on se souvient que la force d'une association est toute contenue dans sa capacité à « transformer des énergies individuelles en capacité d'action collective » (Laville \& Sainsaulieu, 1997). Mais, de fait, AIF doit en permanence imaginer et concevoir des espaces et des moments d'échanges ${ }^{17}$, si ce n'est originaux, à tout le moins adaptés à l'histoire de l'ONG, permettant ainsi de faire tenir ensemble des acteurs aux motivations et logiques d'action différentes, dans un contexte où la dimension émotionnelle est omniprésente.

\section{Bibliographie}

Becker, H.S. (1960). Notes sur le concept d'engagement. Tracés. Revue de Sciences humaines, 11, 2006 ( $1^{\mathrm{e}}$ parution 1960). Doi : https://doi.org/10.4000/traces.257

17 Entre autres moments, AIF a aussi mis en place des Rencontres interrégionales, un Campus d'été, des Rencontres Jeunes qui complètent la structure organisationnelle bâtie autour des Groupes locaux, des Antennes jeunes, du Conseil national, du Secrétariat national et de l'Assemblée générale. 
Bernard, F. (2015). La communication des organisations entre questions d'influence et questions d'autonomie. L'actualité des notions d'engagement, d'émergence et d'institution. Communication et organisation, 47, 85-95. Doi : https://doi.org/10.4000/ communicationorganisation.4909

Bernardeau Moreau, D. \& Hély, M. (2007). Transformations et inerties du bénévolat associatif sur la période 1982-2002. Sociologies pratiques, 15(2), 9-23. Doi : https:// doi.org/10.3917/sopr.015.0009

Béroud, S. (2013). Perspectives critiques sur la participation dans le monde du travail : éléments de repérage et de discussion. Participations, 5(1), 5-32. Doi : https://doi. org/10.3917/parti.005.0005

Blanchet, A. \& Gotman, A. (2006). L'Enquête et ses méthodes : l'entretien. Paris : Armand Colin.

Bloch-Lainé, F. (1963). Pour une réforme de l'entreprise. Paris : Seuil.

Bonnafous, S. \& Krieg-Planque, A. (2013). L'analyse du discours. Dans S. Olivesi (dir.), Sciences de l'information et de la communication. Objets, savoirs, discipline (pp. 223-238). Coll. Communication en +. Grenoble : Presses universitaires de Grenoble.

Buccolo, É., Eynaud, P. \& Haeringer, J. (2014). La Gouvernance des associations en pratiques (étude). Paris : Le Mouvement associatif. Disponible à : https://www.alsacemouvementassociatif.org/index.php/guides-pratiques/55-autres-guides/297-lagouvernance-des-associations-en-pratique

Cefaï, D., Carrel, M., Talpin, J., Eliasoph, N. \& Lichterman, P. (2012). Ethnographies de la participation. Participations, 4(3), 7-48. Doi : https://doi.org/10.3917/parti.004.0005

Combes, M.-C. \& Ughetto, P. (2010). Malaise dans l'association : travail, organisation et engagement. Travailler, 24(2), 153-174. Doi : https://doi.org/10.3917/ trav.024.0153

Demoustier, D. (2002). Le bénévolat, du militantisme au volontariat. Revue française des affaires sociales, 4, 97-116. Doi : https://doi.org/10.3917/rfas.024.0097

Ferreras, I. (2007). Critique politique du travail. Travailler à l'heure de la société des services. Paris : Sciences Po Les Presses.

Giroux, N. \& Giordano, Y. (1998). Les deux conceptions de la communication du changement. Revue française de gestion, 26-27, septembre-octobre, 139-151.

Hachour, H. (2011). Épistémologies socio-sémiotiques et communication organisante : la coproduction de sens comme moteur de l'organisation. Communication et organisation, 39, 195-210. Doi : https://doi.org/10.4000/communicationorganisation.3139

Havard-Duclos, B. \& Nicourd, S (2005). Le bénévolat n'est pas le résultat d'une volonté individuelle. Pensée plurielle, 9(1), 61-73. Doi : https://doi.org/10.3917/ pp.009.0061

Hély, M. (2010). Le travail « d'utilité sociale » dans le monde associatif. Management \& Avenir, 40/10, 206-217. https://doi.org/10.3917/mav.040.0206

Hély, M. \& Simonet, M. (éds) (2013). Le Travail associatif. Paris : Presses universitaires de Paris Ouest.

Hirschman, A.O. (1970). Exit, voice, loyalty. Défection et prise de parole. Bruxelles : Éditions de l'Université de Bruxelles, 1995 (1éd. 1970).

Ion, J. (1997). La Fin des militants ? Coll. Enjeux de société. Paris : Les Éditions de l'Atelier. 
Joule, R.-V. \& Beauvois, J.-L. (1987). Petit traité de manipulation à l'usage des honnêtes gens. Grenoble: Presses universitaires de Grenoble, 2002 (1 ${ }^{\mathrm{e}}$ éd. 1987).

Lacoste, M. (2000). L'espace du langage. De l'accomplissement du travail à son organisation. Sciences de la société, 50 \& 51, 197-216.

Laville, J.-L. (2013). L'association, articulation difficile entre un projet et une organisation. Les Idées en mouvement, août-septembre.

Laville, J.-L. \& Sainsaulieu, R. (Éd.) (1997). Sociologie de l'association. Des organisations à l'épreuve du changement social. Bruxelles : Desclée de Brouwer.

de Montalembert, A. (1980). Amnesty International. La défense des droits de l'homme à la portée de tous. Études, 352(3), 319-332. Doi : https://doi.org/10.3917/ etu. 523.0319

Nicourd, S. (2007). Les engagements ont-ils vraiment changé ? Sociologies pratiques, 15(2), 1-5. Doi : https://doi.org/10.3917/sopr.015.0001

Pierron, J.-P. (2006). L'engagement : envies d'agir, raisons d'agir. Sens-dessous, 0/1, 51-61. Doi : https://doi.org/10.3917/sdes.000.0051

Reynaud, J.-D. (1993). Les Règles du jeu. L'action collective et la régulation sociale. Coll. U. Paris : Armand Colin, 2004 (1éd. 1993).

Seurrat, A. (2014). Écrire un mémoire en sciences de l'information et de la communication. Récits de cas, démarches et méthodes. Coll. Les fondamentaux de la Sorbonne Nouvelle. Paris : Presses Sorbonne Nouvelle.

Simonet, M. (2006). Chapitre 11 - Le monde associatif : entre travail et engagement. Dans N. Alter (Éd.), Sociologie du monde du travail (pp. 191-208). Paris : PUF.

Vermeersch, S. (2004). Entre individualisation et participation : l'engagement associatif bénévole. Revue française de sociologie, 45(4), 681-710. Doi : https://doi. org/10.3917/rfs.454.0681 


\section{(c) (1) $(9)$}

BY NC ND Publié sous la licence Creative Common

«Attibution - pas d'utilisation Commerciale - Pas de Modification 4.0. International» (CC BY-NC-ND) 